\title{
Review of: "Reconstruction of sound driven, actively amplified and spontaneous motions within the tree cricket auditory organ"
}

\author{
Ole Næsbye Larsen ${ }^{1}$ \\ 1 University of Southern Denmark
}

Potential competing interests: The author(s) declared that no potential competing interests exist.

Earlier this year Vavakou et al. (2021) introduced the relatively new vibration measuring technique OCT (Optical Coherence Tomography) to investigate the critical link between sound reception by eardrums (tympana) and activation of auditory receptor cells in a bush cricket, an orthopteran insect of the ensiferan family Tettigoniidae. Until now such an investigation had been impossible as the widely used 45-years old technique of laser Doppler vibrometry is limited to surface measurements and often involves the placement of a reflecting bead on the vibrating structure, whereas OCT allows almost non-invasive depthresolved measurements.

The present, highly interesting manuscript by Mhatre et al. extends the study of the link between sound reception by tympana and activation of auditory receptor cells to another ensiferan family, the Gryllidae, where in tree crickets mechanosensory cells are known to generate spontaneous oscillations and amplify sound-evoked vibrations - much like in vertebrates. This study takes the OCT-technique a step further using VOCT (Volumetric Optical Coherence Tomography), which allows high-resolution simultaneous identification of anatomical surface and subsurface structures and of their vibration patterns (amplitude and phase).

The study reveals and quantifies several important aspects of the micromechanics of the tree cricket ear, especially the relative vibrational trajectories of surface and subsurface ear components and the role of active amplification from supposedly actively bending dendritic cilia of the tympanal organ, which is mechanically coupled to the tracheal wall. With the new technique this model system holds promise of revealing not only further aspects of the micromechanics of ensiferan ears but also of general principles of audition.

Specific minor comments:

Abstract. The word "trachea". Absolutely correct but I suggest that you indicate the plural (two tracheal branches) by the Latin "tracheae" or the English "tracheas".

Introduction. Very clear and exemplary Introduction. But please insert a reference to Vavakou et al. (2021) in the fourth paragraph together with (Dewey et al., 2019; Lee et al., 2015).

Results. 3D structure including Fig. 1. Very clear description. However, your choice of axis designation initially confused me as I would have expected the Z- and Y-axes to be swapped such that Fig. 1C would 
show an X-Y plane, whereas the Z-axis would indicate the long axis of the tibia. Please insert $X$ and $Z$ indicators in Fig. 1C to make your choice of axis designation immediately clear to slow readers like me. In the figure legend (Fig. 1E), I suggest that you include relevant references to the Drosophila studies. Results. 2D vibrometric measurements including Fig. 2. In the third paragraph you use the reference pressure 2e-5 Nm-2, which is correct, but I suggest instead to use "20 $\mu \mathrm{Pa}$ ", which is more familiar to bioacousticians (and you also use pascals ( $\mathrm{Pa}$ ) on the ordinate of Fig. 3 and Suppl. Fig. 1). In the fourth paragraph, I find the use of the word "vertical" a bit confusing as in the normal standing position this direction would be horizontal - or did I misunderstand? I recommend that you stick to the X-Y-Z directions throughout the manuscript.

Results. Single point vibrometric measurements including Fig. 3. In the second paragraph you write “... treated as independent variables and the independent variable was sensitivity or displacement per unit sound pressure level." Shouldn't it be “... the dependent variable was sensitivity ..."? In the fourth paragraph, how do you arrive at $85.53 \%$ ? $(73.0 \%-9.48 \%) / 73.0 \%=0.87$. Also, can you really justify the four-digit result? It seems overly precise to me. I would accept $86 \%$. Finally, I am rather confused when comparing the statements in paragraph five and six. In paragraph five you state that at $40 \mathrm{~dB}$ the sensitivities of atymp and tracheal wall in the z-direction were not significantly different. In paragraph six, however, you state that "this pattern was also maintained" at $80 \mathrm{~dB}$ but now write "The sensitivity of the external anterior tympanal membrane in the $z$ direction was significantly lower $9.48 \pm 4.23 \mathrm{~nm} / \mathrm{Pa}$ but that of the internal tracheal wall was not significantly different at $7.65 \pm 3.95 \mathrm{~nm} / \mathrm{Pa}$." Lower than what? Different from what? Is this the same pattern as at $40 \mathrm{~dB}$ ? Please clarify.

Results. Reconstruction of auditory organ motion including Fig. 4. In the second paragraph, I do not see any reference to Fig. 4A and 4B. Shouldn't they be referred to in this paragraph in addition to Fig. 3D? They are very informative. In the third paragraph, you use the expression "vertical and lateral". Again, I suggest that you stick to your coordinate designations using expressions like "in the $z$ - and x-directions". The suggestion that the cilium undergoes a bending movement in addition to its shortening is highly interesting and deserves further study.

Discussion. Comprehensive, fine, and satisfying. Personally, I always wondered why the cricket main leg trachea splits into two at the tympanal organ. Could it be that the dividing wall between the two compartments is needed to create the elliptical path of the internal attachment point illustrated in Fig. 4C and 4D?

Methods. Since VOCT is not familiar to most readers, I suggest that you include a supplementary figure with a sketch of the animal in the setup with its foreleg in the laser beam and the placement of speaker and microphone. 